\title{
ARTICLE
}

\section{Retrospective dosimetry using TL/OSL method}

\author{
David Mesterhazy ${ }^{*}$, Margit Osvay, Andras Kovacs and Andras Kelemen \\ Centre for Energy Research, Hungarian Academy of Science, 1121 Budapest, Hungary
}

\begin{abstract}
The increasing risk of violent terror attacks concerning radioactive or nuclear materials require preparatory investigations. Environmental disasters and unexpected events prove also the requirement of different analytical methods which are able to moderate the impact on human race. After these events suitable dose estimation is required within the less time possible. Several methods and processes have been developed in the last few years for retrospective determination or estimation of dose conditions in the affected area. Electronic devices such as SMD resistors have been investigated and results show that suitable sensitivity and dose response verify the use of the method. This paper discusses further and detailed investigations of luminescence properties of SMD resistors and the usage of them in real emergency.
\end{abstract}

Keywords: retrospective dosimetry; thermoluminescence; optically stimulated luminescence; ceramics

\section{Introduction}

Luminescent dosimetry is a widely-used and reliable method not just in personal, but environmental and retrospective dosimetry as well. Thermoluminescence and optically stimulated luminescence are similar light emitting processes, well-known and investigated.

Although the handling of nuclear materials have become more secure the risk of a not peaceful usage increases and denotes danger to the human race. Environmental disasters also influence scientific investigations and force researchers to develop subservient methods in emergency situation.

After an unexpected event involving nuclear or radioactive materials the most seriously affected people are those who have been in the area at the moment of explosion or emission. This means that arrangements have to be made before the event to be sure that the consequences could be the less harmful as possible.

Lots of methods are considered as possibilities for retrospective estimation of received dose, however few of them are suitable in real emergency as well regarding the field application. Investigations have also proved that lots of materials and personal objects can be applied for retrospective dosimetry purposes such as telephone chip-cards etc.[1-3].

Our earlier investigations have shown that TL measurements of SMD electronic devices seem to be reliable method for retrospective dosimetry purposes. This further study shows detailed characteristics of the electronic SMD parts, emphasizing those, which are critical considering the real usage conditions.

The aim of this study is to prove the reliability of the

*Corresponding author. Email: mesterhazydavid@gmail.com method and to make the dose estimation possible. Linearity, fading properties and the effect of the preheating will be represented in this paper. The potential of OSL measurements have also been shown earlier [4] accordingly the study of the combined TL/OSL method will also be presented.

\section{Materials and methods}

The object of the experiments were various commercially available electronic components called Surface Mount Devices (SMD). Earlier SMD resonators have also been investigated, but in the interval of interest (0 Gy - 5 Gy) their sensitivity using TL method was not adequate. Nine commercially available SMD resistors have been chosen for the experiments which are widely used in different electronic devices. SMD resistors are shown in Figure 1.

The main component of SMD resistors is $\mathrm{Al}_{2} \mathrm{O}_{3}$ (more than 90\%) and $\mathrm{SiO}_{2}$ [5]. Both of them are well-known for luminescent properties and sensitivity.

TL measurements have been carried out using Daybreak photon-counting reader having a heating tray and PM assembly with an EMI 9635QA tube (quartz window). Temperature of heating cycle reached $300^{\circ} \mathrm{C}$; the heating rate during evaluation was $4{ }^{\circ} \mathrm{C} / \mathrm{s}$. Annealing of the samples between irradiations was made using a furnace. Besides measurements SMD resistors were kept in container to exclude the undesirable light exposures.

For TL measurements the samples were given different doses in the range of $90 \mathrm{mGy}-1440 \mathrm{mGy}$ using a calibrated ${ }^{90} \mathrm{Sr}-{ }^{90} \mathrm{Y}$ reference dose irradiator (Studsvik 6527 B). 


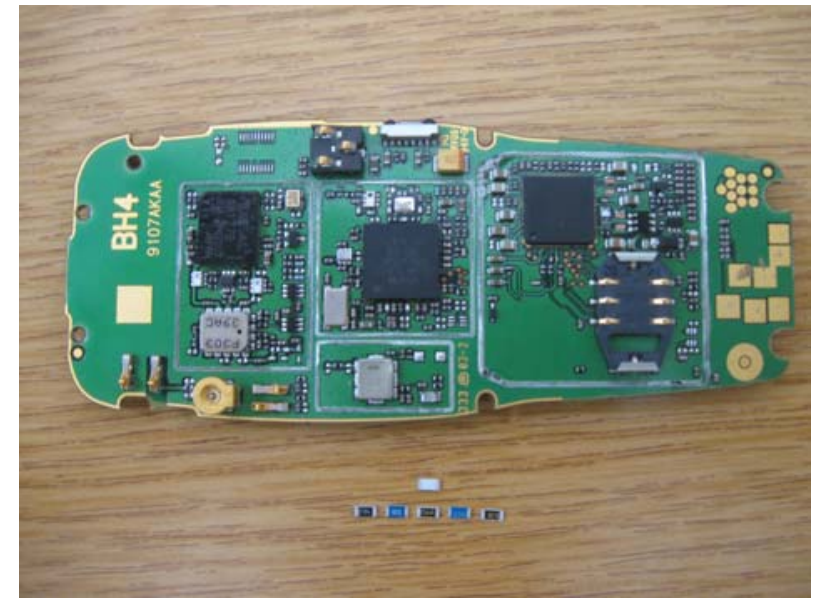

Figure 1. SMD resistors from electronic devices.

OSL measurements were carried out in the Geological Institute of Hungary. The four major parts of the Risø DA-20 are: a) EMI 9235 QA bialcali photomultiplier, b) sample heater part, c) optical stimulation part (49 LED), d) $1.48 \mathrm{GBq}$ activity ${ }^{90} \mathrm{Sr}-$ ${ }^{90} \mathrm{Y}$ source. Continuous wave OSL and Linearly Modulated OSL measurements can be carried out using the device.

\section{Results and discussion}

\subsection{Thermoluminescence}

\subsubsection{Linearity}

Dose-response is one of the most important properties of TL materials as well as other materials used for dosimetry purposes. With an increasing dose increasing response is expected, sometimes it approximates a linear increase.

Nine SMD resistors have been annealed for 30 minutes and have been irradiated using the following doses: $90 \mathrm{mGy}, 180 \mathrm{mGy}, 360 \mathrm{mGy}, 720 \mathrm{mGy}$, and $1440 \mathrm{mGy}$. Net areas of the received glow-curves have been calculated. Figure 2 shows the TL response of the nine SMD resistors versus the received dose.

Though curves are not linear observing the entire dose range, in different intervals the increasing response is represented. The response of the resistors is similar, the difference among them is not an order of magnitude. Some of them show proportional increment in lower below $400 \mathrm{mGy}$ in our case - doses but few are proportional in higher doses. Although the sensitivity of $150 \mathrm{k} \Omega \mathrm{SMD}$ is the lowest the signal increment is consistent in this case.

\subsubsection{Fading}

Thermoluminescent dosimeter's signal decreases after irradiation with time, this effect is called fading [6]. In real emergency situation fast dose estimation is required within the least time possible. That necessitates previously defined fading properties to be able to prove that received data approaches or equals to the real received dose.

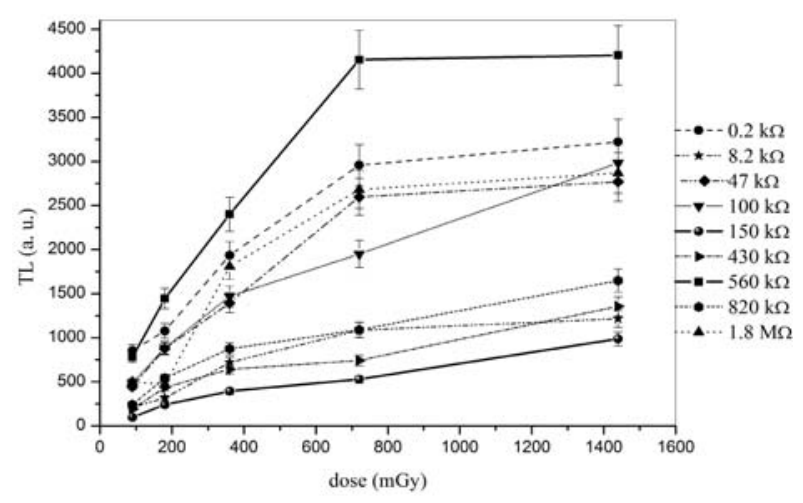

Figure 2. Dose response of SMD resistors.

Fading properties have been determined by irradiating the nine selected SMD resistors and storing them in darkness. A series of samples have been evaluated directly after the irradiation called zero. Further measurements have been done after 1 day, 2 days, 5 days and 8 days respectively. Figure 3 shows the fading properties of SMD resistors.

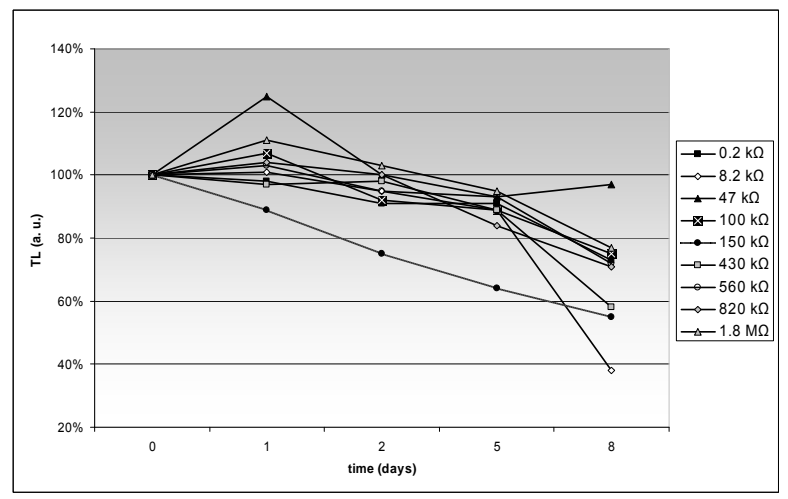

Figure 3. Fading of the SMD resistors (\%).

\subsubsection{Post-irradiation preheat}

Annealing is very important in thermoluminescence dosimetry to rely on results. Standard annealing procedures are given to each material known and commercially produced. Standard deviation and reliability of results are only guaranteed if the process is done before usage of the dosimeters. This means annealing is very important step of evaluation and influences the results. Post-irradiation preheat is done to remove lower satellite peaks and clarify suitable signal. It standardizes glow curves and ensures the reproducibility.

Investigations have been done after normal annealing process. A series of SMD resistors have been evaluated just after irradiation, three more sets using post-irradiation preheating with $60^{\circ} \mathrm{C}, 80^{\circ} \mathrm{C}$, and $100{ }^{\circ} \mathrm{C}$. Figure 4 shows the final results represented in the same diagram. 


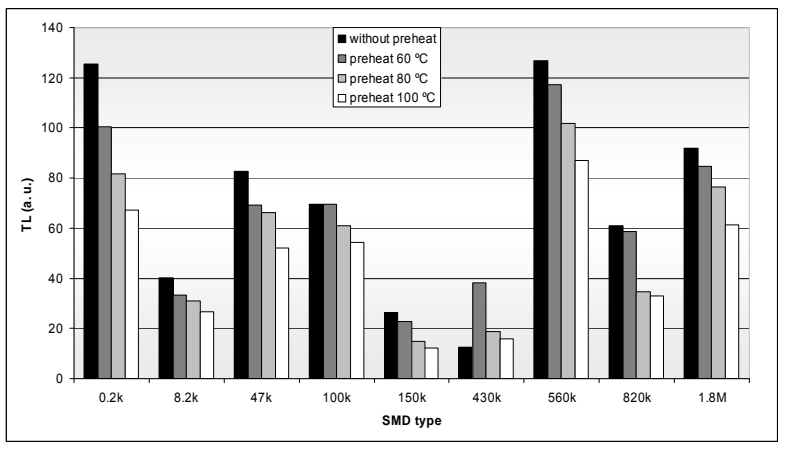

Figure 4. Effect of post-irradiation preheating on glow curves of SMD resistors.

The decline of TL intensity has been expected in advance and Figure 4 shows a progressive decline in almost all the cases. Though $430 \mathrm{k} \Omega$ type SMD is different than others - although its TL intensity is the lowest in this case - the decreasing tendency of the resistors is very similar. It can be concluded that the shape of the glow curves do not change a lot neither do the net area values.

\subsection{Optically stimulated luminescence}

\subsubsection{Dose response}

First step of evaluation was to set the adequate preheat temperature using a preliminary determined dose (1.431 Gy). The result has shown that using $140{ }^{\circ} \mathrm{C}$ can be reached to the best reproducibility. The obtaining of the growth curve (Figure 5) was done using this preheat process.

Figure 5 shows the steadily increasing OSL signal of SMD resistor. Although the increment is lower in higher doses - saturation of traps - the signal is close to be linear in lower dose ranges - below $10-20$ Gy dose in that case. Consequently Figure 5 contains the enlarged inset of the lower dose range as well to show the difference from higher doses. To prove the favourable character of the OSL signal linear fit is shown in the diagram with red.

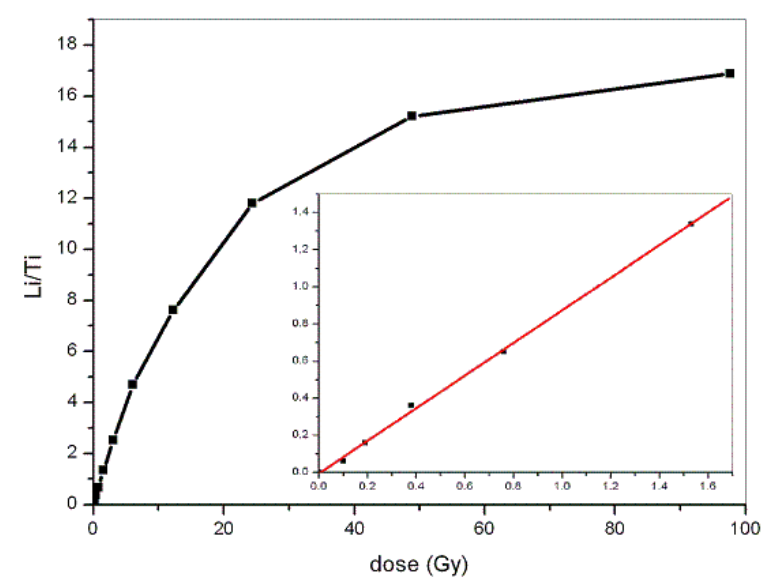

Figure 5. OSL growth curve of SMD resistor in the dose range of $0.1 \mathrm{~Gy}-97.7 \mathrm{~Gy}$ (lower dose range enlarged).

\section{Conclusion}

Widely used SMD resistors from different electronic devices have been investigated using TL and OSL method. Experiments have been carried out to prove the reliability of the method. Different parameters of SMD resistors have been investigated and as result have become given.

In this paper SMD resistors have been characterized and the results are the following. It has been shown that the investigated resistors have suitable TL signal in the selected dose range (dose response, fading properties and $8 \%$ previously determined standard deviation). However the increasing TL signal is not linear but approaches to that. It can also be concluded that their fading properties are acceptable for retrospective dosimetry purposes. Investigation of post-irradiation annealing has given the result that glow curves - and along with this net area as well - do not change substantially. That means if the preliminary expected dose values are not very low, an annealing process of $100{ }^{\circ} \mathrm{C}$ could be applied. Finally OSL results show that the increasing signal of an SMD resistor is suitable and in lower dose ranges it can be considered as linear undoubtedly.

\section{Acknowledgements}

We would like to thank to Thamóné Bozsó Edit (Geological Institute of Hungary) for the possibility and the continuous support in the optically stimulated luminescence measurements.

\section{References}

[1] H. Y. Göksu, Telephone chip-cards as individual dosimeters, Radiat. Meas. 37 (2003), pp. 617-620.

[2] E. L. Inrig, D. I. Godfrey-Smith and S. Khanna, Optically stimulated luminescence of electronic components for forensic, retrospective and accidental dosimetry, Radiat. Meas. 43 (2008), pp. 726-730.

[3] K. Beerten, C. Woda and F. Vanhavere, Thermoluminescence dosimetry of electronic components for forensic, retrospective and accident dosimetry, Radiat. Meas. 44 (2009), pp. 620-625.

[4] E. G. Yukihara, R. Gaza, S. W. S. McKeever and C. G. Soares, Optically stimulated luminescence and thermoluminescence efficiencies for high-energy heavy charged particle irradiation in $\mathrm{Al}_{2} \mathrm{O}_{3}: \mathrm{C}$, Radiat. Meas. 38 (2004), pp. 59-70.

[5] D. Ekendahl and L. Judas, Retrospective dosimetry with alumina substrate from electronic components, Radiat. Prot. Dosim. 150 (2012), pp. 134-141.

[6] S. W. S. McKeever, M. Moscovitch and P. D. Townsend, Thermoluminescence Dosimetry Materials: Properties and Uses, Nuclear Technology Publishing, 1995, Asford, ISBN 1870965191. 